\title{
Cellular Automata based Encrypted ECG-hash Code Generation: An Application in Inter-human Biometric Authentication System
}

\author{
Subrata Nandi, Satyabrata Roy, Jayanti Dansana \\ Computer Science and Engineering Department, KIIT University, Bhubaneswar, 751024, India \\ Email: \{subrotoster, satya2k6ster, jayantidansana486\}@ gmail.com
}

\begin{abstract}
Wahiba Ben Abdessalem Karaa
Computer Science and Engineering Department, High Institute of Management, 41, Rue de la Liberté, Cité Bouchoucha 2000 Le Bardo, Tunis, TUNISIA

Email: wahiba.abdessalem@isg.rnu.tn
\end{abstract}

\begin{abstract}
Ruben Ray
Department of IT, Government College of Engineering and Leather Technology, Kolkata, 700098, India

Email: ruben.ray@gmail.com
\end{abstract}

\author{
Shatadru Roy Chowdhury, Sayan Chakraborty, Nilanjan Dey \\ Department of CSE, JIS College of Engineering, Kalyani, 741235, India \\ Email: shatadru_jcs10@yahoo.in, sayan.cb@gmail.com, neelanjan.dey@gmail.com
}

\begin{abstract}
In this modern era, biometrics incorporate various mechanisms to recognize inimitable features of human beings by utilizing their biological and evident features. This paper proposes a novel technique for constructing a resilient and secure biometric recognition system. In this paper, an ECG-hash code of two distinct individuals has been formed by taking dot product of electrocardiogram (ECG) feature matrices of two persons located at two different sites at respective databases. The validity of the system increases as samples from both persons, between whom the transmission takes place, are essential. Besides, electrocardiogram is such a unique feature of an individual that could not be compromised at any circumstance as contradictory to other features like fingerprints, face recognition etc. Moreover, the ECGhash code is encrypted using rule vector of cellular automata that gives better security in terms of randomness of generated cipher text.
\end{abstract}

Index Terms - Inimitable, electrocardiogram, dot product, cellular automata, ECG-hash code, randomness, cipher text.

\section{INTRODUCTION}

This era is characterized by expeditious growth in terms of communication and exchange of information over wireless channels that tend to be highly insecure. It gives rise to an authentication mechanism so that operational data and/or electronic transactions do not go in wrong hands unintentionally. The essence to authenticate the receiver on the other hand is very crucial for preserving integrity and confidentiality of the sent information. Capacity of human brain falls short in memorizing passwords and/or IDs that are among the most popular way of authentication now-a-days. Besides, due to the erroneous practice of human beings, sometimes they forget to carry those credentials or these credentials may be stolen or copied. So, biometric devices that use unique traits of individuals like fingerprints, face recognition, retina scan etc. have started to become useful as they use inherent features of a person. But these systems have certain limitations. When a person ages or gets some injuries, then these types of systems tend to fail giving faulty outcome. But ECG and EEG are among the features that survive from these limitations due to their inherent mechanism. The process of biometric authentication consists of two steps - a) enrollment and b) verification [19]. In enrollment step, a template is produced after taking out the required features from an individual's biometric data. Verification process is the way of generating a new template from a user's biometric data after a raw scan and matching it with the previous one [1]. The person is authentic if and only if the value of essential parameters after comparison lies within a previously defined threshold value.

In general, there are two ways to achieve biometric authentication e.g. uni-modal [2], [3] and multi-modal [4], [5]. False acceptance rate (FAR) and false rejection rate (FRR) are the two parameters that measure the performance of a biometric system [1]. Equal error rate (ERR) is when these two parameters become same. Mathematically, $\mathrm{ERR}=(\mathrm{FAR}+\mathrm{FRR}) / 2$. A biometric system is perfect when the value of ERR is zero [6]. As mentioned above, biometric systems have some 
limitations. When it goes to an intruder [4], data privacy is violated. A person's retina and fingerprints changes with time. Solution of the problem is cancelable biometrics [5]. Cancelable biometrics is more secure as it is non invertible and it can be reused [1]. So it does not demand to be kept secret [7]. But this non invertible transform is not said to be non-vulnerable. This gives birth to bio-hash, which is also a form of cancelable biometrics [8]. This bio-hash is obtained by performing dot product of the biometric feature of a person in some iterations and a tokenized random number provided by user. This is the process of extracting feature matrix from an individual's biometric feature. So it provides better security as both the random number and the particular biometric feature is required to crack it. Moreover, this system has zero error rate point [ERP] [8].

\section{MOTIVATION}

In this work, electrocardiogram (ECG) has been used as a biometric feature due to its uniqueness.

ECG is a tool to diagnose the presence of any abnormality in the heart of any individual and is based upon the electrical activities of heart. ECG is unique and depends on physical configuration especially that of the chest of any individual. Therefore it can be used to uniquely identify any person. A standard ECG for a single cardiac cycle is shown in Fig. 1.

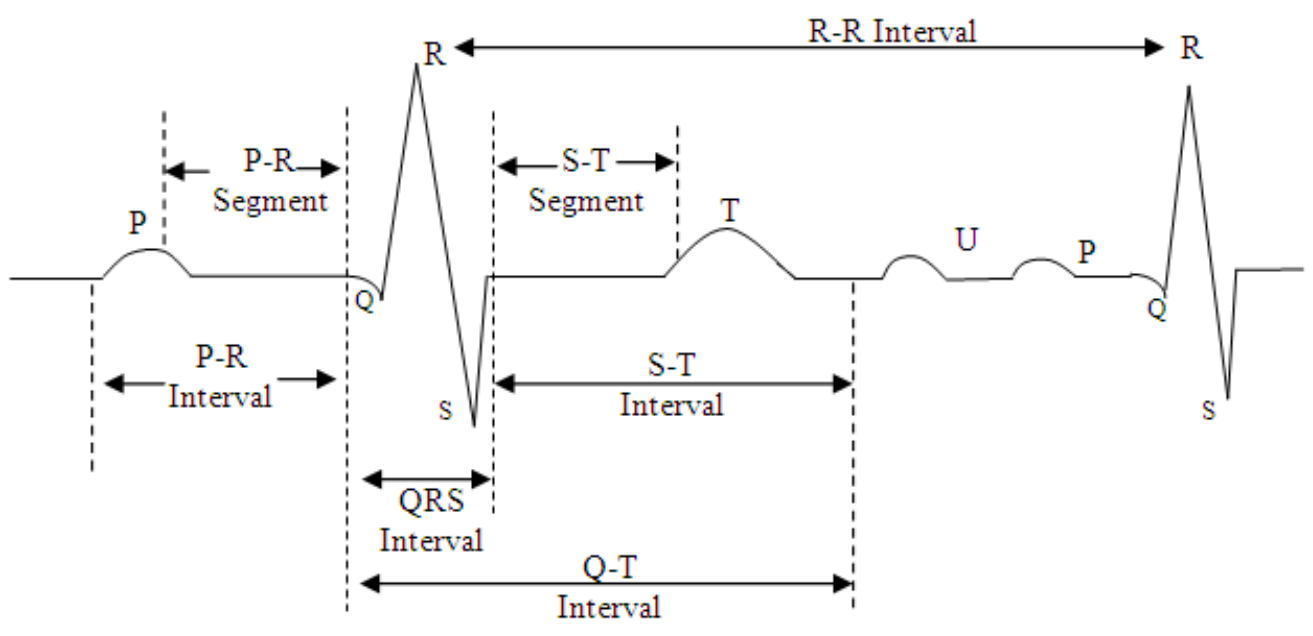

Fig.1. P-QRS-T complex detected ECG signal [1]

Fig. 1 contains a P-wave, a QRS complex, a T -wave and a U-wave [8].

In this current work, a dot product is performed between ECG feature matrices, obtained from two different individuals, from two remote sites. The use of more than one characteristic in multimodal approach does not stop any user from being authenticated even if one trait fails because the others are still used to match. But, the major limitation is that the characteristics obtained are from a single entity. There are approaches where the biometric sample is obtained from two individuals [1]. Since ECG feature is unique and it is obtained from two individuals, it gives better security than multimodal approach. In this paper one more security issue has been added. The newly generated ECG-hash code is further encrypted using rule vectors of cellular automata.

Recent studies suggest that many characteristics of cellular automata can be used efficiently to achieve essential cryptographic criteria i.e. balanced, correlation immune, non-linearity etc. CA can also be used to generate balanced and high quality rules that consist of various radiuses [9] and to generate pseudo random number [10]. CA can also be used to generate random cipher text in symmetric key cryptographic purpose as reported in [11]. Here, the same technique as of [11] is applied to encrypt the ECG-hash code before transmission. This gives more security because of three issues: a) ECG signal [20] is unique b) biometric feature matrix is obtained from two individuals and c) the ECGhash code is encrypted using $\mathrm{CA}$ rule vector before transmission.

\section{BASIC OF CELlular AutomatA}

\section{A. Cellular Automata}

Cellular automata are an array of cells capable of storing one bit at a time. Each cell transits into a new state at next timestamp depending on the states of its neighbors and a transition rule [11]. So cellular automata can be defined by three tuples $(\mathrm{S}, \mathrm{T}, \mathrm{N})$, where $\mathrm{S}$ is the non empty finite set of states, $\mathrm{T}$ is the non empty finite set of transition rules, and $\mathrm{N}$ is the non empty finite set of neighbors. CA was invented by J. v. Neumann [12] and was popularized by S. Wolfram [13]. For example, consider 2 states, 3 neighborhood one dimensional CA. The first row represents all possible cell values of three cells at timestamp t. Next row onwards represent the state of the current cell at timestamp $t+1$. Here we consider current cell as $i$, the left hand neighbor as i-1 and right hand neighbor as $\mathrm{i}+1$. 
Table 1. Example rules for next state update

\begin{tabular}{|c|c|c|c|c|c|c|c|c|}
\hline $\begin{array}{c}\text { Rule } \\
\text { No }\end{array}$ & 111 & 110 & 101 & 100 & 011 & 010 & 001 & 000 \\
\hline 51 & 0 & 0 & 1 & 1 & 0 & 0 & 1 & 1 \\
\hline 60 & 0 & 0 & 1 & 1 & 1 & 1 & 0 & 0 \\
\hline 102 & 0 & 1 & 1 & 0 & 0 & 1 & 1 & 0 \\
\hline 153 & 1 & 0 & 0 & 1 & 1 & 0 & 0 & 1 \\
\hline 195 & 1 & 1 & 0 & 0 & 0 & 0 & 1 & 1 \\
\hline
\end{tabular}

$\mathrm{T}$ is conventionally represented as characteristic matrix of CA that is composed of rules of all cells. It is an $n \times n$ (for $n$ cells) matrix constructed according to the rule for individual cell. $\mathrm{j}$-th row stands for a rule applicable to the $\mathrm{j}$-th cell. If its next state is based on a particular cell, then its position in matrix $\mathrm{T}$ is marked by ' 1 ', otherwise it is marked by ' 0 '. The state transition is mathematically represented as: $\left[P_{t+1}(x)\right]=[T] \times\left[P_{t}(x)\right]$, where $\mathrm{P}_{\mathrm{t}+1}(\mathrm{x})$ is the state of cell $\mathrm{i}$ at $\mathrm{t}+1$ timestamp and $\mathrm{P}_{\mathrm{t}}(\mathrm{x})$ is the state of cell $i$ at timestamp t. The rules can be represented as follows:

$$
\begin{aligned}
& \text { Rule 51: } P_{t+1}(x)=\overline{P_{t}(x)} \\
& \text { Rule 60: } P_{t+1}(x)=P_{t}(x) \oplus P_{t}(x-1) \\
& \text { Rule 102: } P_{t+1}(x)=P_{t}(x+1) \oplus P_{t}(x) \\
& \text { Rule 153: } P_{t+1}(x)=\overline{P_{t}(x+1) \oplus P_{t}(x)} \\
& \text { Rule 195: } P_{t+1}(x)=\overline{P_{t}(x) \oplus P_{t}(x-1)}
\end{aligned}
$$

There are total 256 such rules of one dimensional cellular automaton [11]. The CAs can be of many types viz. additive, non-additive, periodic boundary, null boundary, programmable CA, Group CA etc. as reported in [14].

\section{B. Group Cellular Automata}

A cellular automaton is called group cellular automata when it regenerates the initial bit string after a certain number of iteration after application of a particular rule vector. Mathematically,

$$
\begin{aligned}
& {[T]^{n}=I(I \text { is the identity matrix })} \\
& {\left[P_{t+n}(x)\right]=[T]^{n} \times\left[P_{t}(x)\right]}
\end{aligned}
$$

where, $\mathrm{n}$ denotes the order of the group[15].

We can have 256 combinations when rule 51 and 102 are applied [11]. Out of these 256 combinations, $<11000011>$ is preferred where 1 denotes rule 51 and 0 denotes rule 102. The order of the group cellular automata used in this work is 8. So, ECG-hash code is encrypted in 4 iterations using the above rule vector and applying the same vector, in next 4 iterations the encrypted ECG-hash code is decrypted.

\section{PROPOSED SYSTEM}

The block diagram of the proposed system is given below:

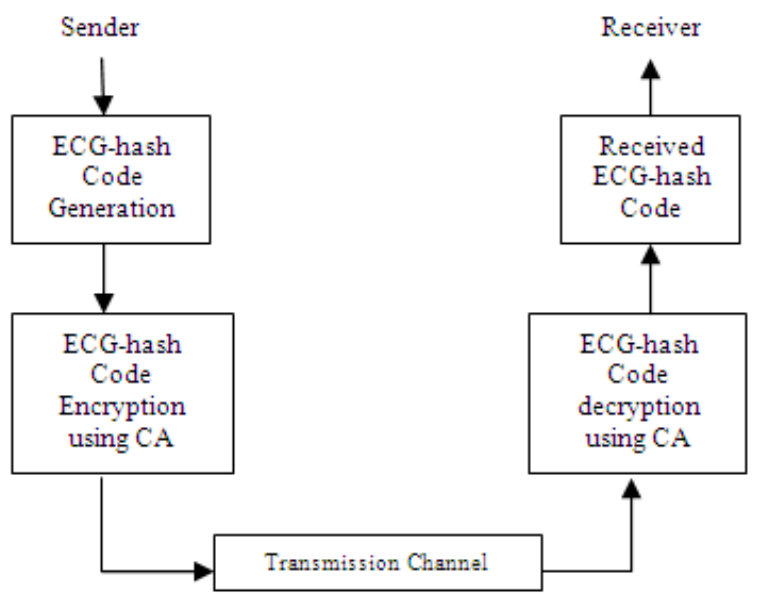

Fig.2. Block diagram of overall system

\section{A. Generation of ECG-hash Code}

Step 1. Modified Pan-Tompkin's algorithm [16][17] is applied on ECG signal to detect P, QRS, and T components.

Step 2. ECG features like R-R, P-P, Q-Q, S-S, P-R, and $\mathrm{Q}-\mathrm{T}$ is measured from the ECG signal whose peaks are detected.

Step 3. A matrix of dimension $8 \times 32$ is generated based on the features detected from ECG.

Step 4. Dot product is performed between two matrices, one of sender and another of receiver, that is stored in the sender's database (DB1).

Step 5. The product is compared with the threshold value set beforehand/a priori. If the value exceeds the threshold then set this to binary 1 otherwise it is reset to 0 .

Step 6. The newly generated ECG-hash code is sent to CA encryption system.

\section{B. Encryption of ECG-hash Code using CA}

The input to this encryption system is a binary ECGhash code of length 32 . We divide this into four 8-bit blocks. Then the following algorithm is applied.

Step 1. An 8-bit block is fed into programmable cellular automata.

Step 2. A rule vector $<11000011>$ is applied to each cell, where 1 represents $\mathrm{CA}$ rule 51 and 0 represents CA rule 102.

Step 3. The PCA is run for four consecutive clock cycles.

Step 4. Encrypted plaintext or cipher text is generated.

Step 5. Step 1 to step 4 is repeated until remaining 3 blocks are encrypted.

Below is the block diagram of generating encrypted ECG-hash code. 


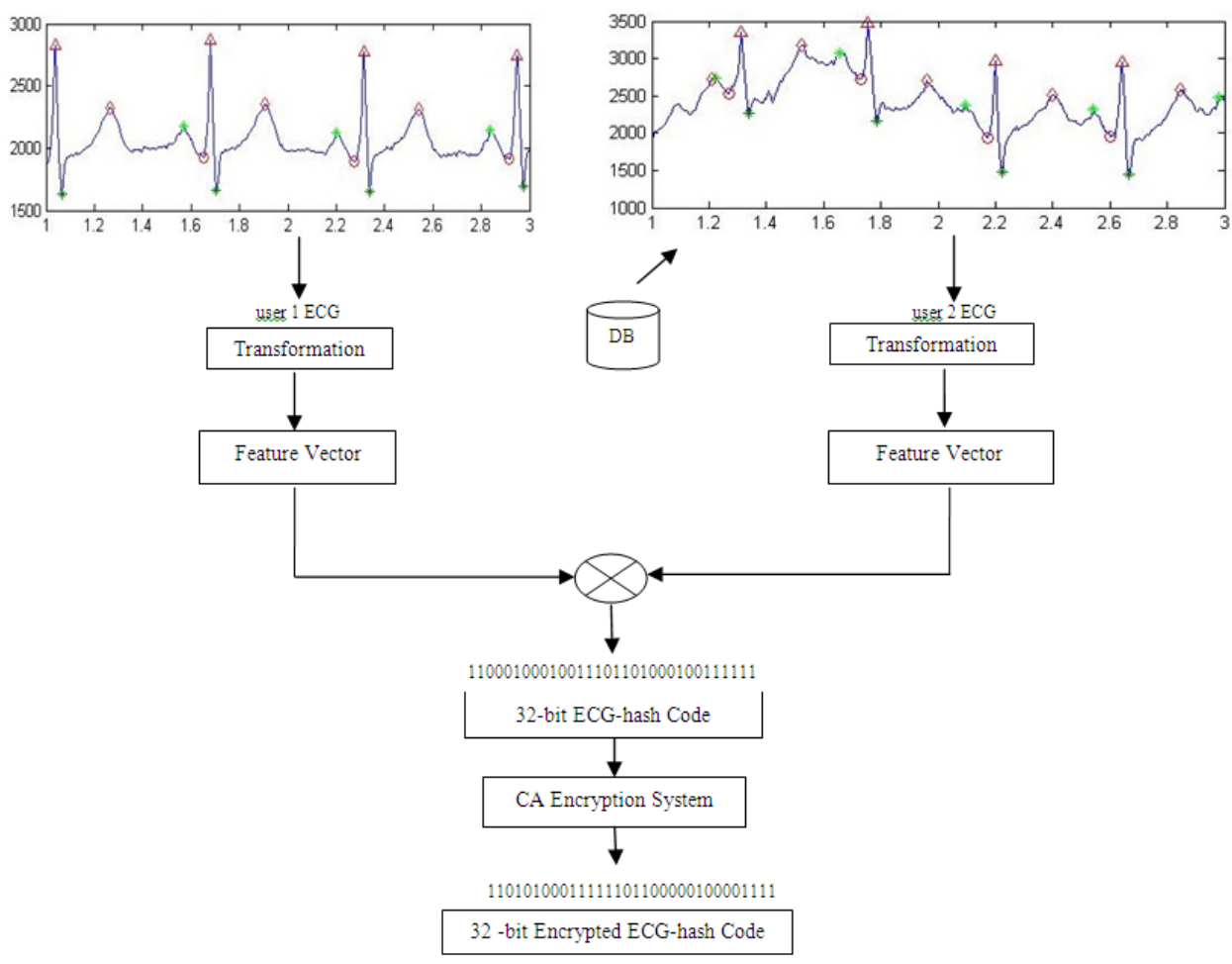

Fig.3. Encrypted Hash Code Generation

\section{Decryption of encoded ECG-hash code on Receiver}

Input to this encryption system is the encrypted binary ECG-hash code of length 32 . We divide this also into four 8-bit blocks. Then following algorithm is applied.

Step 1. A single 8 bit block is fed into programmable cellular automata.

Step 2. A rule vector $<11000011>$ is applied to each cell, where 1 represents $\mathrm{CA}$ rule 51 and 0 represents CA rule 102.

Step 3. The PCA is run for four consecutive clock cycles.

Step 4. Encrypted plaintext or cipher text is generated.

Step 5. Step 1 to step 4 is repeated until remaining 3 blocks are encrypted.

Fig. 4 is the block diagram of the decryption of ECGhash code using $\mathrm{CA}$.

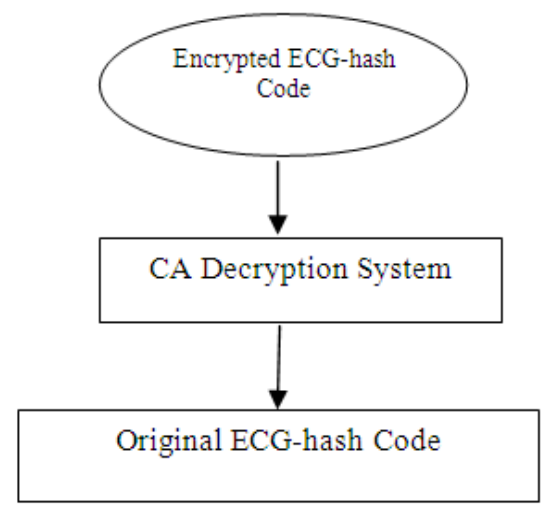

Fig.4. Decryption of encrypted ECG-hash Code
Fig. 5 below describes the encryption of first 8 bits of 32bit ECG-hash code using cellular automata rule vector $<11000011>$ as mentioned in the algorithm.

$\begin{array}{cccccccc}<51 & 51 & 102 & 102 & 102 & 102 & 51 & 51> \\ 1 & 1 & 0 & 0 & 0 & 1 & 0 & 0 \\ 0 & 0 & 0 & 0 & 1 & 1 & 1 & 1 \\ 1 & 1 & 0 & 1 & 0 & 0 & 0 & 0 \\ 0 & 0 & 1 & 1 & 0 & 0 & 1 & 1 \\ 1 & 1 & 0 & 1 & 0 & 1 & 0 & 0\end{array}$

Fig. 5. Encryption of the first 8 bits of generated 32-bit ECG-hash Code

Fig. 6 below depicts the decryption of first 8 bits of 32 bit ECG-hash code.

$\begin{array}{cccccccc}<51 & 51 & 102 & 102 & 102 & 102 & 5 & 51> \\ 1 & 1 & 0 & 1 & 0 & 1 & 0 & 0 \\ 0 & 0 & 1 & 1 & 1 & 1 & 1 & 1 \\ 1 & 1 & 0 & 0 & 0 & 0 & 0 & 0 \\ 0 & 0 & 0 & 0 & 0 & 0 & 1 & 1 \\ 1 & 1 & 0 & 0 & 0 & 1 & 0 & 0\end{array}$

Fig. 6. Decryption of first 8 bits of 32 bit ECG-hash Code 


\section{RESULTS}

In this work, we have used Matlab 7.0.1 to perform ECG-hash code generation and for comparative analysis purpose. We have used Dev C++ 4.9.9.2 to apply cellular automata encryption and decryption algorithm and obtaining result. In table 2 through 5 , we have tabulated the time duration for P-P, -Q, R-R, T-T, P-R, Q-T, and QTc, ORS of user 1.

Table 2. P-P and Q-Q time intervals

\begin{tabular}{|c|c|c|c|c|}
\hline Sl. No. & Interval & $\begin{array}{c}\text { Time } \\
\text { Intervals } \\
\text { (sec) }\end{array}$ & Interval & $\begin{array}{c}\text { Time } \\
\text { Intervals } \\
\text { (sec) }\end{array}$ \\
\hline 1 & \multirow{32}{*}{ P-P } & 0.6400 & \multirow{32}{*}{ Q-Q } & 0.6300 \\
\hline 2 & & 0.6450 & & 0.6550 \\
\hline 3 & & 0.6300 & & 0.6250 \\
\hline 4 & & 0.6350 & & 0.6400 \\
\hline 5 & & 0.6250 & & 0.6000 \\
\hline 6 & & 0.6200 & & 0.6500 \\
\hline 7 & & 0.6250 & & 0.6150 \\
\hline 8 & & 0.6000 & & 0.6000 \\
\hline 9 & & 0.6200 & & 0.6000 \\
\hline 10 & & 0.6050 & & 0.6350 \\
\hline 11 & & 0.6250 & & 0.6250 \\
\hline 12 & & 0.6250 & & 0.6000 \\
\hline 13 & & 0.6300 & & 0.6450 \\
\hline 14 & & 0.6500 & & 0.6200 \\
\hline 15 & & 0.6300 & & 0.6550 \\
\hline 16 & & 0.6200 & & 0.6050 \\
\hline 17 & & 0.6250 & & 0.6250 \\
\hline 18 & & 0.6200 & & 0.6400 \\
\hline 19 & & 0.6300 & & 0.6150 \\
\hline 20 & & 0.6300 & & 0.6250 \\
\hline 21 & & 0.6050 & & 0.6050 \\
\hline 22 & & 0.6300 & & 0.6350 \\
\hline 23 & & 0.6400 & & 0.6300 \\
\hline 24 & & 0.6300 & & 0.6500 \\
\hline 25 & & 0.3300 & & 0.2600 \\
\hline 26 & & 0.4200 & & 0.5000 \\
\hline 27 & & 0.6400 & & 0.6300 \\
\hline 28 & & 0.6450 & & 0.6550 \\
\hline 29 & & 0.6300 & & 0.6250 \\
\hline 30 & & 0.6350 & & 0.6400 \\
\hline 31 & & 0.6250 & & 0.6000 \\
\hline 32 & & 0.6200 & & 0.6500 \\
\hline
\end{tabular}

Table 3. R-R and T-T time intervals

\begin{tabular}{|c|c|c|c|c|}
\hline $\begin{array}{c}\text { Sl. } \\
\text { No. }\end{array}$ & Interval & $\begin{array}{c}\text { Time } \\
\text { Intervals } \\
\text { (sec) }\end{array}$ & Interval & $\begin{array}{c}\text { Time } \\
\text { Intervals } \\
\text { (sec) }\end{array}$ \\
\hline 1 & \multirow{32}{*}{ R-R } & 0.6450 & \multirow{32}{*}{$\mathrm{T}-\mathrm{T}$} & 0.6450 \\
\hline 2 & & 0.6400 & & 0.6400 \\
\hline 3 & & 0.6350 & & 0.6350 \\
\hline 4 & & 0.6300 & & 0.6400 \\
\hline 5 & & 0.6250 & & 0.6200 \\
\hline 6 & & 0.6250 & & 0.6200 \\
\hline 7 & & 0.6150 & & 0.6100 \\
\hline 8 & & 0.6100 & & 0.6100 \\
\hline 9 & & 0.6150 & & 0.6200 \\
\hline 10 & & 0.6100 & & 0.6100 \\
\hline 11 & & 0.6250 & & 0.6200 \\
\hline 12 & & 0.6250 & & 0.6350 \\
\hline 13 & & 0.6250 & & 0.6300 \\
\hline 14 & & 0.6450 & & 0.6350 \\
\hline 15 & & 0.6350 & & 0.6400 \\
\hline 16 & & 0.6250 & & 0.6200 \\
\hline 17 & & 0.6200 & & 0.6200 \\
\hline 18 & & 0.6200 & & 0.6250 \\
\hline 19 & & 0.6250 & & 0.6250 \\
\hline 20 & & 0.6250 & & 0.6250 \\
\hline 21 & & 0.6200 & & 0.6100 \\
\hline 22 & & 0.6250 & & 0.6300 \\
\hline 23 & & 0.6300 & & 0.6300 \\
\hline 24 & & 0.6400 & & 0.6350 \\
\hline 25 & & 0.2250 & & 0.1700 \\
\hline 26 & & 0.5250 & & 0.5850 \\
\hline 27 & & 0.6450 & & 0.6450 \\
\hline 28 & & 0.6400 & & 0.6400 \\
\hline 29 & & 0.6350 & & 0.6350 \\
\hline 30 & & 0.6300 & & 0.6400 \\
\hline 31 & & 0.6250 & & 0.6200 \\
\hline 32 & & 0.6250 & & 0.6200 \\
\hline
\end{tabular}


Table 4. P-R and Q-T time intervals

\begin{tabular}{|c|c|c|c|c|}
\hline $\begin{array}{l}\text { Sl. } \\
\text { No. }\end{array}$ & Interval & $\begin{array}{c}\text { Time } \\
\text { Intervals } \\
\text { (sec) }\end{array}$ & Interval & $\begin{array}{c}\text { Time } \\
\text { Intervals } \\
\text { (sec) }\end{array}$ \\
\hline 1 & \multirow{32}{*}{ P-R } & 0.1100 & \multirow{32}{*}{ Q-T } & 0.2550 \\
\hline 2 & & 0.1150 & & 0.2700 \\
\hline 3 & & 0.1100 & & 0.2550 \\
\hline 4 & & 0.1150 & & 0.2650 \\
\hline 5 & & 0.1100 & & 0.2650 \\
\hline 6 & & 0.1100 & & 0.2850 \\
\hline 7 & & 0.1150 & & 0.2550 \\
\hline 8 & & 0.1050 & & 0.2500 \\
\hline 9 & & 0.1150 & & 0.2600 \\
\hline 10 & & 0.1100 & & 0.2800 \\
\hline 11 & & 0.1150 & & 0.2550 \\
\hline 12 & & 0.1150 & & 0.2500 \\
\hline 13 & & 0.1150 & & 0.2850 \\
\hline 14 & & 0.1100 & & 0.2700 \\
\hline 15 & & 0.1050 & & 0.2850 \\
\hline 16 & & 0.1100 & & 0.2700 \\
\hline 17 & & 0.1150 & & 0.2850 \\
\hline 18 & & 0.1100 & & 0.2800 \\
\hline 19 & & 0.1100 & & 0.2650 \\
\hline 20 & & 0.1050 & & 0.2750 \\
\hline 21 & & 0.1000 & & 0.2750 \\
\hline 22 & & 0.1150 & & 0.2800 \\
\hline 23 & & 0.1100 & & 0.2750 \\
\hline 24 & & 0.1000 & & 0.2750 \\
\hline 25 & & 0.1100 & & 0.2600 \\
\hline 26 & & 0.0050 & & 0.1700 \\
\hline 27 & & 0.1100 & & 0.2550 \\
\hline 28 & & 0.1150 & & 0.2700 \\
\hline 29 & & 0.1100 & & 0.2550 \\
\hline 30 & & 0.1150 & & 0.2650 \\
\hline 31 & & 0.1100 & & 0.2650 \\
\hline 32 & & 0.1100 & & 0.2850 \\
\hline
\end{tabular}

Table 5. Q-Tc and QRS time intervals

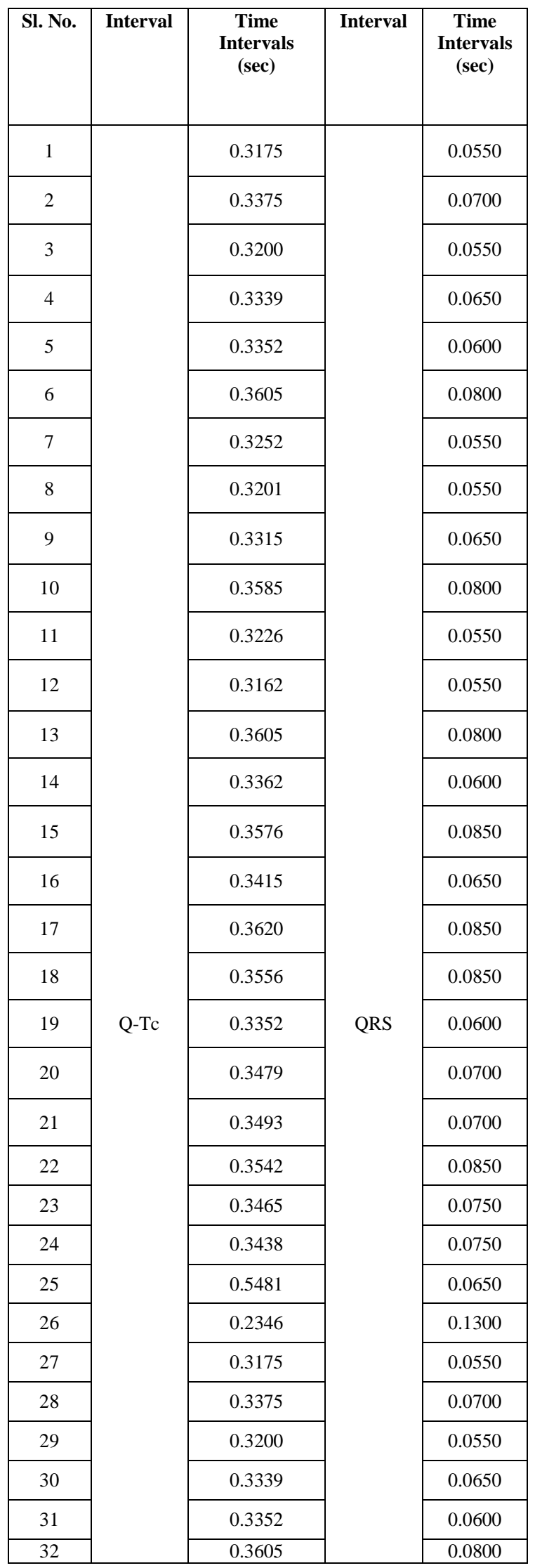


In table 6 through 9, we have tabulated the time duration for P-P, -Q, R-R, T-T, P-R, Q-T, and Q-Tc, ORS of user 2 .

Table 6. P-P and Q-Q time intervals

\begin{tabular}{|c|c|c|c|c|}
\hline $\begin{array}{l}\text { Sl. } \\
\text { No. }\end{array}$ & Interval & $\begin{array}{c}\text { Time } \\
\text { Intervals } \\
\text { (sec) }\end{array}$ & Interval & $\begin{array}{c}\text { Time } \\
\text { Intervals } \\
\text { (sec) }\end{array}$ \\
\hline 1 & \multirow{32}{*}{ P-P } & 0.4200 & \multirow{32}{*}{ Q-Q } & 0.3850 \\
\hline 2 & & 0.4900 & & 0.5200 \\
\hline 3 & & 0.4300 & & 0.4600 \\
\hline 4 & & 0.4400 & & 0.4450 \\
\hline 5 & & 0.4450 & & 0.4250 \\
\hline 6 & & 0.4400 & & 0.4400 \\
\hline 7 & & 0.4600 & & 0.4600 \\
\hline 8 & & 0.4300 & & 0.4300 \\
\hline 9 & & 0.4400 & & 0.4500 \\
\hline 10 & & 0.4500 & & 0.4600 \\
\hline 11 & & 0.4500 & & 0.4450 \\
\hline 12 & & 0.4500 & & 0.4500 \\
\hline 13 & & 0.4450 & & 0.4300 \\
\hline 14 & & 0.4500 & & 0.4650 \\
\hline 15 & & 0.4650 & & 0.4550 \\
\hline 16 & & 0.4500 & & 0.4450 \\
\hline 17 & & 0.4550 & & 0.4700 \\
\hline 18 & & 0.4550 & & 0.4550 \\
\hline 19 & & 0.4550 & & 0.4350 \\
\hline 20 & & 0.4650 & & 0.4650 \\
\hline 21 & & 0.4300 & & 0.4500 \\
\hline 22 & & 0.4450 & & 0.4450 \\
\hline 23 & & 0.4400 & & 0.4400 \\
\hline 24 & & 0.4550 & & 0.4350 \\
\hline 25 & & 0.4400 & & 0.4450 \\
\hline 26 & & 0.4400 & & 0.4400 \\
\hline 27 & & 0.4500 & & 0.4550 \\
\hline 28 & & 0.4550 & & 0.4500 \\
\hline 29 & & 0.4350 & & 0.4450 \\
\hline 30 & & 0.4700 & & 0.4450 \\
\hline 31 & & 0.4350 & & 0.4650 \\
\hline 32 & & 0.4550 & & 0.4450 \\
\hline
\end{tabular}

Table 7. R-R and T-T time intervals

\begin{tabular}{|c|c|c|c|c|}
\hline $\begin{array}{l}\text { Sl. } \\
\text { No. }\end{array}$ & Interval & $\begin{array}{c}\text { Time } \\
\text { Intervals } \\
\text { (sec) }\end{array}$ & Interval & $\begin{array}{c}\text { Time } \\
\text { Intervals } \\
\text { (sec) }\end{array}$ \\
\hline 1 & \multirow{32}{*}{ R-R } & 0.4500 & \multirow{32}{*}{$\mathrm{T}-\mathrm{T}$} & 0.5900 \\
\hline 2 & & 0.4500 & & 0.3150 \\
\hline 3 & & 0.4450 & & 0.4350 \\
\hline 4 & & 0.4450 & & 0.4400 \\
\hline 5 & & 0.4450 & & 0.4450 \\
\hline 6 & & 0.4400 & & 0.4600 \\
\hline 7 & & 0.4450 & & 0.4250 \\
\hline 8 & & 0.4450 & & 0.4650 \\
\hline 9 & & 0.4400 & & 0.4400 \\
\hline 10 & & 0.4500 & & 0.4450 \\
\hline 11 & & 0.4500 & & 0.4450 \\
\hline 12 & & 0.4450 & & 0.4450 \\
\hline 13 & & 0.4500 & & 0.4500 \\
\hline 14 & & 0.4500 & & 0.4500 \\
\hline 15 & & 0.4500 & & 0.4450 \\
\hline 16 & & 0.4600 & & 0.4600 \\
\hline 17 & & 0.4550 & & 0.4600 \\
\hline 18 & & 0.4550 & & 0.4450 \\
\hline 19 & & 0.4550 & & 0.4650 \\
\hline 20 & & 0.4500 & & 0.4500 \\
\hline 21 & & 0.4450 & & 0.4400 \\
\hline 22 & & 0.4500 & & 0.4500 \\
\hline 23 & & 0.4450 & & 0.4500 \\
\hline 24 & & 0.4450 & & 0.4400 \\
\hline 25 & & 0.4400 & & 0.4450 \\
\hline 26 & & 0.4450 & & 0.4450 \\
\hline 27 & & 0.4450 & & 0.4400 \\
\hline 28 & & 0.4450 & & 0.4450 \\
\hline 29 & & 0.4500 & & 0.4500 \\
\hline 30 & & 0.4550 & & 0.4500 \\
\hline 31 & & 0.4500 & & 0.4550 \\
\hline 32 & & 0.4500 & & 0.4500 \\
\hline
\end{tabular}


Table 8. P-R and Q-T time intervals

\begin{tabular}{|c|c|c|c|c|}
\hline $\begin{array}{l}\text { Sl. } \\
\text { No. }\end{array}$ & Interval & $\begin{array}{c}\text { Time } \\
\text { Intervals } \\
\text { (sec) }\end{array}$ & Interval & $\begin{array}{c}\text { Time } \\
\text { Intervals } \\
\text { (sec) }\end{array}$ \\
\hline 1 & \multirow{32}{*}{ P-R } & 0.0950 & \multirow{32}{*}{ Q-T } & 0.2550 \\
\hline 2 & & 0.1250 & & 0.4600 \\
\hline 3 & & 0.0850 & & 0.2550 \\
\hline 4 & & 0.1000 & & 0.2300 \\
\hline 5 & & 0.1050 & & 0.2250 \\
\hline 6 & & 0.1050 & & 0.2450 \\
\hline 7 & & 0.1050 & & 0.2650 \\
\hline 8 & & 0.0900 & & 0.2300 \\
\hline 9 & & 0.1050 & & 0.2650 \\
\hline 10 & & 0.1050 & & 0.2550 \\
\hline 11 & & 0.1050 & & 0.2400 \\
\hline 12 & & 0.1050 & & 0.2400 \\
\hline 13 & & 0.1000 & & 0.2350 \\
\hline 14 & & 0.1050 & & 0.2550 \\
\hline 15 & & 0.1050 & & 0.2400 \\
\hline 16 & & 0.0900 & & 0.2300 \\
\hline 17 & & 0.1000 & & 0.2450 \\
\hline 18 & & 0.1000 & & 0.2350 \\
\hline 19 & & 0.1000 & & 0.2250 \\
\hline 20 & & 0.1000 & & 0.2550 \\
\hline 21 & & 0.0850 & & 0.2400 \\
\hline 22 & & 0.1000 & & 0.2300 \\
\hline 23 & & 0.1050 & & 0.2350 \\
\hline 24 & & 0.1100 & & 0.2450 \\
\hline 25 & & 0.1000 & & 0.2500 \\
\hline 26 & & 0.1000 & & 0.2500 \\
\hline 27 & & 0.1050 & & 0.2550 \\
\hline 28 & & 0.1000 & & 0.2400 \\
\hline 29 & & 0.0900 & & 0.2350 \\
\hline 30 & & 0.1050 & & 0.2400 \\
\hline 31 & & 0.0900 & & 0.2450 \\
\hline 32 & & 0.1050 & & 0.2350 \\
\hline
\end{tabular}

Table 9. Q-Tc and QRS time intervals

\begin{tabular}{|c|c|c|c|c|}
\hline $\begin{array}{l}\text { Sl. } \\
\text { No. }\end{array}$ & Interval & $\begin{array}{c}\text { Time } \\
\text { Intervals } \\
\text { (sec) }\end{array}$ & Interval & $\begin{array}{c}\text { Time } \\
\text { Intervals } \\
\text { (sec) }\end{array}$ \\
\hline 1 & \multirow{40}{*}{ Q-Tc } & 0.3801 & \multirow{40}{*}{ QRS } & 0.0700 \\
\hline 2 & & 0.6857 & & 0 \\
\hline 3 & & 0.3823 & & 0.0700 \\
\hline 4 & & 0.3448 & & 0.0550 \\
\hline 5 & & 0.3373 & & 0.0500 \\
\hline 6 & & 0.3694 & & 0.0650 \\
\hline 7 & & 0.3973 & & 0.0700 \\
\hline 8 & & 0.3448 & & 0.0550 \\
\hline 9 & & 0.3995 & & 0.0700 \\
\hline 10 & & 0.3801 & & 0.0650 \\
\hline 11 & & 0.3578 & & 0.0500 \\
\hline 12 & & 0.3598 & & 0.0550 \\
\hline 13 & & 0.3503 & & 0.0500 \\
\hline 14 & & 0.3801 & & 0.0700 \\
\hline 15 & & 0.3578 & & 0.0550 \\
\hline 16 & & 0.3391 & & 0.0600 \\
\hline 9 & & 0.3995 & & 0.0700 \\
\hline 10 & & 0.3801 & & 0.0650 \\
\hline 11 & & 0.3578 & & 0.0500 \\
\hline 12 & & 0.3598 & & 0.0550 \\
\hline 13 & & 0.3503 & & 0.0500 \\
\hline 14 & & 0.3801 & & 0.0700 \\
\hline 15 & & 0.3578 & & 0.0550 \\
\hline 16 & & 0.3391 & & 0.0600 \\
\hline 17 & & 0.3632 & & 0.0650 \\
\hline 18 & & 0.3484 & & 0.0500 \\
\hline 19 & & 0.3336 & & 0.0500 \\
\hline 20 & & 0.3801 & & 0.0700 \\
\hline 21 & & 0.3598 & & 0.0550 \\
\hline 22 & & 0.3429 & & 0.0500 \\
\hline 23 & & 0.3523 & & 0.0500 \\
\hline 24 & & 0.3673 & & 0.0550 \\
\hline 25 & & 0.3769 & & 0.0700 \\
\hline 26 & & 0.3748 & & 0.0700 \\
\hline 27 & & 0.3823 & & 0.0750 \\
\hline 28 & & 0.3598 & & 0.0600 \\
\hline 29 & & 0.3503 & & 0.0550 \\
\hline 30 & & 0.3558 & & 0.0650 \\
\hline 31 & & 0.3652 & & 0.0700 \\
\hline 32 & & 0.3503 & & 0.0550 \\
\hline
\end{tabular}


Now for user 1: The means of selected attributes for consecutive peaks are: $\operatorname{Mean}_{1}=1.3468, \operatorname{Mean}_{2}=1.3293$, Mean $_{3}=1.3322$, Mean $_{4}=1.2238$ etc.; where each mean is considered as the threshold value $(\mathrm{T})$ for its respective block.

We have got first block of the Keymatrix ${ }_{1}$ as shown below:

$\begin{array}{llllllll}0.6400 & 0.6450 & 0.6300 & 0.6350 & 0.6250 & 0.6200 & 0.6250 & 0.6000 \\ 0.6300 & 0.6550 & 0.6300 & 0.6250 & 0.6400 & 0.6000 & 0.6500 & 0.6150 \\ 0.6450 & 0.6400 & 0.6350 & 0.6300 & 0.6250 & 0.6250 & 0.6150 & 0.6100 \\ 0.6450 & 0.6400 & 0.6350 & 0.6400 & 0.6200 & 0.6200 & 0.6100 & 0.6100 \\ 0.1100 & 0.1150 & 0.1100 & 0.1150 & 0.1100 & 0.1100 & 0.1150 & 0.1050 \\ 0.2550 & 0.2700 & 0.2550 & 0.2650 & 0.2650 & 0.2850 & 0.2550 & 0.2500 \\ 0.3175 & 0.3375 & 0.3200 & 0.3339 & 0.3352 & 0.3605 & 0.3252 & 0.3201 \\ 0.0550 & 0.0700 & 0.0550 & 0.0650 & 0.0600 & 0.0800 & 0.0550 & 0.0550\end{array}$

Fig.7. Keymatrix 1

Similarly, we have got the first block of the Keymatrix $_{2}$ as shown below:

$\begin{array}{cccccccc}0.4200 & 0.4900 & 0.4300 & 0.4400 & 0.4450 & 0.4400 & 0.4600 & 0.4300 \\ 0.3850 & 0.5200 & 0.4600 & 0.4450 & 0.4250 & 0.4400 & 0.4600 & 0.4300 \\ 0.4500 & 0.4500 & 0.4450 & 0.4450 & 0.4450 & 0.4400 & 0.4450 & 0.4450 \\ 0.5900 & 0.3150 & 0.4350 & 0.4400 & 0.4450 & 0.4600 & 0.4250 & 0.4650 \\ 0.0950 & 0.1250 & 0.0850 & 0.1000 & 0.1050 & 0.1050 & 0.1050 & 0.0900 \\ 0.2550 & 0.4600 & 0.2550 & 0.2300 & 0.2250 & 0.2450 & 0.2650 & 0.2300 \\ 0.3801 & 0.6857 & 0.3823 & 0.3448 & 0.3373 & 0.3694 & 0.3973 & 0.3448 \\ 0.0700 & 0 & 0.0700 & 0.0550 & 0.0500 & 0.0650 & 0.0700 & 0.0550\end{array}$

Fig.8. Keymatrix2

Original and cropped ECG signal from user 1 and user 2 are shown in the Fig. 8 below:
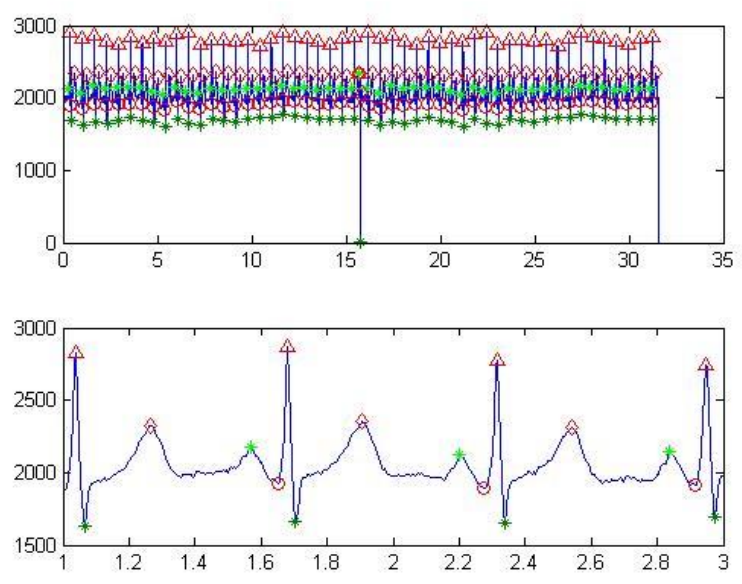

Fig.8. Signal and peak detection of user 1
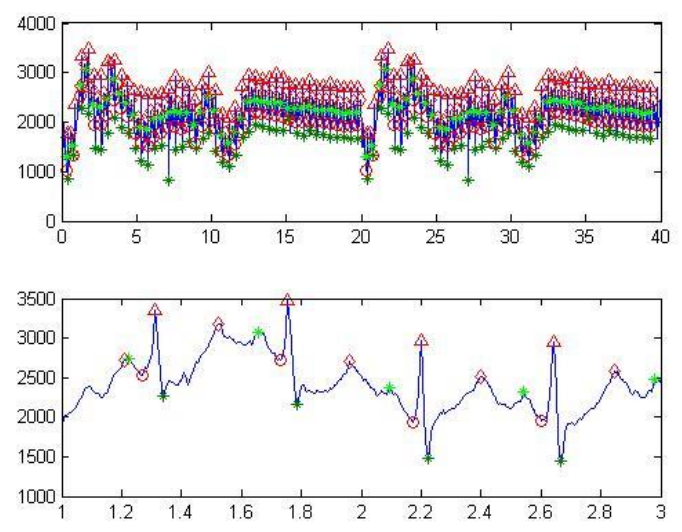

Fig.9. Signal and peak detection of user 2

Here is how we have gone through the process of extraction of feature matrix. There are total of four 8-bit blocks of Keymatrix ${ }_{1}$ and Keymatrix K $_{2}$.

\section{From User 1}

Block $B_{1}$ $\mathrm{Block} \mathrm{B}_{4}$

0.64000 .64500 .63000 .63500 .62500 .62000 .62500 .6000
0.63000 .65500 .63000 .62500 .64000 .60000 .65000 .6150
0.64500 .64000 .63500 .63000 .62500 .62500 .61500 .6100
0.64500 .64000 .63500 .64000 .62000 .62000 .61000 .6100
0.11000 .11500 .11000 .11500 .11000 .11000 .11500 .1050
0.25500 .27000 .25500 .26500 .26500 .28500 .25500 .2500
0.31750 .33750 .32000 .33390 .33520 .36050 .32520 .3201
0.05500 .07000 .05500 .06500 .06000 .8000 .05500 .0550

From User 2

Block B' ${ }_{1}$

Block B' 4

0.42000 .49000 .43000 .44000 .44500 .44000 .46000 .4300

0.38500 .52000 .46000 .44500 .42500 .44000 .46000 .4300

0.45000 .45000 .44500 .44500 .44500 .44000 .44500 .4450

0.59000 .31500 .43500 .44000 .44500 .46000 .42500 .4650

0.09500 .12500 .08500 .10000 .10500 .10500 .10500 .0900

0.25500 .46000 .25500 .23000 .22500 .24500 .26500 .2300

0.38010 .68570 .38230 .34480 .33730 .36940 .39730 .3448

$0.0700 \quad 0 \quad 0.07000 .05500 .05000 .06500 .07000 .0550$

Fig.11. Division of feature matrices in blocks

Then we calculate Dot Product of this first of blocks of the two Key matrices i.e. $\mathrm{B}_{1}$ and $\mathrm{B}_{1}{ }_{1}$ to obtain the Dotproduct_1 The threshold value, as mentioned before, for this Dotproduct_1 is: 1.3468 . For each of these values, if it is greater than the mean then we insert a ' 1 ' at that position, otherwise it is a ' 0 '. Hence we get the first set of binary key matrix as: 


$$
\left[\begin{array}{llllllll}
1 & 1 & 0 & 0 & 0 & 1 & 0 & 0
\end{array}\right]
$$

Finally, after computing all four sets of eight iterations i.e. a total of 32 iterations, we get the Final key matrix as:

$\begin{array}{llllllll}1 & 1 & 0 & 0 & 0 & 1 & 0 & 0 \\ 0 & 1 & 0 & 0 & 1 & 1 & 1 & 0 \\ 1 & 1 & 0 & 1 & 0 & 0 & 0 & 1 \\ 0 & 0 & 1 & 1 & 1 & 1 & 1 & 1\end{array}$

Now, here is the result of CA encryption algorithm:

Enter the ECGhash code : 11000100010011101 101000100111111

The First block is: 11000100

The Ascii char for the 1 st block is: -

The Second Block is: 01001110

The Ascii char for the 2nd block is: $\mathrm{N}$

The Third Block is: 11010001

The Ascii char for the 3 rd block is: :

The 4th block is: 00111110

The Ascii char for the 4th block is: ?

The Biohashcode in ascii form is : $-\mathrm{N}=$ ?

00001111

11010000

00110011 (4 rounds encryption of 1 st block)

11010100

The ciphertext is: 11010100

First encrypted char in ascii is: $t$

10010001

01110010

10010101 (4 rounds encryption of 2 nd block)

01111110

The ciphertext is: 01111110

2nd encrypted char in ascii is :

00110010

11010101

00111110 (4 rounds encryption of $3 \mathrm{rd}$ block)

11000001

The ciphertext is: 11000001

3 rd encrypted char in ascii is : $\perp$

11000000

00000011

11000100 (4 rounds encryption of 4 th block)

00001111

The ciphertext is: 00001111

The clock tick is : 36.000000

4 th encrypted char in ascii is:

The encrypted ECGhashcode in ascii form: $t \perp-$ The total output is: $\begin{array}{lllllllllllll}1 & 1 & 0 & 1 & 0 & 1 & 0 & 0 & 0 & 1 & 1 & 1 & 1\end{array}$ $\begin{array}{lllllllllllllllllll}1 & 1 & 0 & 1 & 1 & 0 & 0 & 0 & 0 & 0 & 1 & 0 & 0 & 0 & 0 & 1 & 1 & 1 & 1\end{array}$ $\mathrm{c} 1=17.000000$ (No of 1 in ciphertext block) $\mathrm{c} 0=15.000000$ (No of 0 in ciphertext block)

$Y=14.000000$ (No of change of pattern of $1 \mathrm{~s}$ and $0 \mathrm{~s}$ ) Mean $=15.937500$

Standard Deviation $=2.771202$

Value of normal Variate $Z=-0.699155$
First block contains the plaintext and corresponding ASCII value. Then the plaintext block has been broken into 4 blocks plaintext and their ASCII value. After that the blocks has been converted to corresponding cipher text block using cellular automata rules. Then the 4 blocks of cipher text has been merged to the block of encrypted ECG hash code. In the reverse way we can get the plaintext block using the same cellular automata rule matrix.

\section{Performance Analysis:}

\section{A. Analysis of the Algorithm:}

There are 256 rules in 1D cellular automaton [14]. We have used only 2 rules. There are $2^{\wedge} 8(256)$ combination of the 2 rules. We have used one combination. So the hacker need to test $256^{\wedge} 8$ combination which is impossible in case of brute force attack.

\section{B. Statistical Analysis:}

Statistical analysis is very much required in case of cryptographic algorithm to test the randomness of the cipher text. For this purpose runs test [18] has been performed to check the randomness of the algorithm. We have tested the following result for the data as following table:

Table 6. Runs Test Result

\begin{tabular}{|c|c|}
\hline No. of Bits in Plaintext & Value of $\mathrm{Z}$ \\
\hline 32 & -0.699155 \\
\hline 64 & 1.016917 \\
\hline 128 & 0.025013 \\
\hline 256 & -1.791818 \\
\hline
\end{tabular}

From the above result it is observed that the value of the normal variant $(\mathrm{z})$ is less than 1.96. Obtained value of $\mathrm{Z}$ in our study $(|\mathrm{z}|<1.96)$ implies that the difference between the observed and expected is not significant. Our hypothesis for random cipher text generation is true.

\section{CONCLUSION AND FUTURE WORK}

This work highlights a new aspect of verifying and validating an individual's identity to give him access privilege of sensitive and interesting information. Moreover, by applying an encryption algorithm over the generated ECG-hash code from ECG signal [21, 22], its degree of privacy preservation has increased. The essence of having two person's biometric feature has advantages over the other biometric traits that were used so far.

Current work gives more emphasize on offline ECGhash code generation. The future extension of this work will be to make it an online one. Further, attempt will be to implement this system in a real time environment with some time constraints in number of attempts. 


\section{REFERENCES}

[1] M. Dey, N. Dey, S. K. Mahata, S. Chakraborty, S. Acharjee and A. Das, "Electrocardiogram Feature based Inter-human Biometric Authentication System", International Conference on Electronic Systems, Signal Processing and Computing Technologies, 2014.

[2] S Latifi, N Solayappan, "A Survey of Unimodal Biometric Methods", in International Conference on Security \& Management, SAM, 2006, pp. 57-63.

[3] S. S. Parvinder, I. Kaur, A. Verma, S. Jindal, S. Singh. (2009, Nov.). Biometric Methods and Implementation of Algorithms. International Journal of Electrical \& Electronics Engineering [Online].3,(8),pp.3-8.

[4] S. M. Rahal, H. A. Aboalsamah, K. N. Muteb, "Multimodal Biometric Authentication System - MBAS", in 2nd IEEE Conference on Information and Communication Technologies, 2006, pp. 1026-1030.

[5] M. I. Ahmad, W. L. Woo, S. S. Dlay, "Multimodal biometric fusion at feature level: Face and palm print", in 7th International Symposium on Communication Systems Networks and Digital Signal Processing, 2010, pp. 801805.

[6] P. Lacharme, A. Plateaux. PIN-based cancelable biometrics, GREYC Research lab, Ensicaen - UCBN CNRS [Online].3(2), . Available: http://www.ecole.ensicaen.fr/ lacharme/IJAIT2011.pdf. 2011, pp. 75-79

[7] N. Radhal and S. Karthikeyan, "An Evaluation of Fingerprint Security using Noninvertible Biohash", International Journal of Network Security \& Its Applications (IJNSA), 2011, Vol.3, No.4.

[8] N. Dey, B. Nandi, M. Dey, D. Biswas, A. Das, S. S. Chaudhuri, "Generation of Bio Hash Code from Electrocardiogram Features", in $3^{\text {rd }}$ IEEE International Advance Computing Conference, 2013, pp. 724-728.

[9] F. Maleki, A. Mohades, M. E. Shiri, A. Bijari, " A CA randomizers based on parallem CAs with balanced rules", International conference on Computational Science, ICCS, 2010, pp. 417-425.

[10] L. Kotoulas, D. Tsarouchis, G. Ch. Sirakoulis, I. Andreadis, "1-d cellular automata for pseudo random number generation and its reconfigurable hardware implementation", Proceedings of IEEE international symposium on circuits and systems, 2006.

[11] S. Roy, S. Nandi, J. Dasnasa, P. K. Pattnaik, "Application of cellular automata in symmetric key cryptography", Proceedings of IEEE International Conference on Communication and Signal Processing (ICCSP), 2014.

[12] J. V. Neumann, Theory of Self Reproducing Automata, edited and completed by Burks, A.W. (Ed.), Univ. of Illinois press, London, 1966.

[13] S. Wolfram, A new kind of science, Wolfram Media Inc., ISBN: 1-57955-008-8, 2002.

[14] S. Nandi, B. K. Kar, Pabitra Pal Chaudhuri, "Theory and applications of cellular automata in cryptography", IEEE Transactions on Computers, 1994, 43(12), pp. 1346-1356.

[15] D. Mukhopadhyay, "Design and analysis of cellular automata based cryptographic algorithms", Doctoral thesis, Indian Institute of Technology, Kharagpur, 2007.

[16] N. Dey, S. Mukhopadhyay, A. Das, S. S. Chaudhuri, "Analysis of P-QRS-T Components Modified by Blind Watermarking Technique Within the Electrocardiogram Signal for Authentication in Wireless Telecardiology Using DWT”, in IJIGSP, 2012, vol.4, no.7, pp.33-46.
[17] N. Dey, S. Samanta, X-S Yang, A. Das, and S. S. Chaudhuri, "Optimisation of scaling factors in electrocardiogram signal watermarking using cuckoo search', Int. J. Bio-Inspired Computation, 2013, Vol. 5, No. 5, pp.315-326

[18] S. M. Ross, Introductory Statistics, Second edition, Academic Press, Elsevier, pp. 659-664.

[19] N. Dey, A. B. Roy, A. Das and S. S. Chaudhuri, "Recent Trends in Computer Networks and Distributed Systems Security", published in Springer Berlin / Heidelberg in Communications in Computer and Information Science, 2012, ISBN 978-3-642-34134-2, Volume 335, Part 2, Pages 347-357, DOI: 10.1007/978-3-642-34135-9_35.

[20] N. Dey, S. Biswas , A. B. Roy, A. Das and S.S. Chaudhuri, "Analysis Of Photoplethysmographic Signals Modified by Reversible Watermarking Technique using PredictionError in Wireless Telecardiology", International Conference of Intelligent Infrastructure, 47th Annual National Convention of CSI, 2012, McGraw-Hill Proceeding .

[21] N. Dey, P. Das, A. Das and S.S. Chaudhuri, "DWT-DCTSVD Based Blind Watermarking Technique of Gray Scale Image in Electrooculogram Signal”, International Conference on Intelligent Systems Design and Applications (ISDA-2012), pp. 680-685, 2012.

[22] N. Dey, S. Biswas, P. Das, A. Das, and S. S. Chaudhuri, "Feature Analysis for the Reversible Watermarked Electrooculography Signal using Low Distortion Prediction-error Expansion", 2012 International Conference on Communications, Devices and Intelligent Systems (CODIS), pp.624-627, 2012.

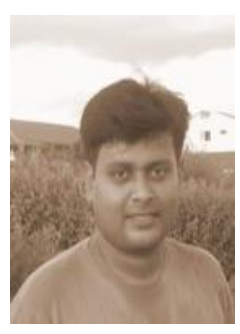

Subrata Nandi, born in 1987, is pursuing M. Tech in Computer Science Engineering from KIIT University, Bhubaneswar, India. His major area in research is cellular automata in cryptography and algorithm design and analysis.

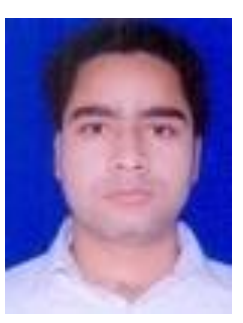

Satyabrata Roy, is pursuing Master of Technology degree from KIIT University, Bhubaneswar, Orissa, India. $\mathrm{He}$ was lecturer in Jaipur Engineering College, Jaipur for 18 months and in Grow More Faculty of Engineering, Gujarat for 18 months. Cellualr automata is his major area of interest. He has three research papers in international conferences.

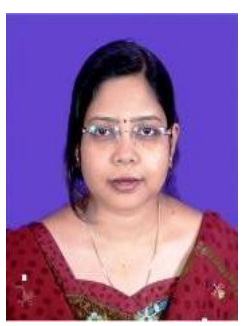

Jayanti Dansana has completed her M.Tech from Utkal University, Odisha in 2008.Currently she is working as Assistant Professor in School Of Computer Engineering, KIIT University, Bhubaneswar, Odisha. Her research area is Data Mining, Distributed System. Her publication included around 5 to 10 papers in the area of Data Mining. 

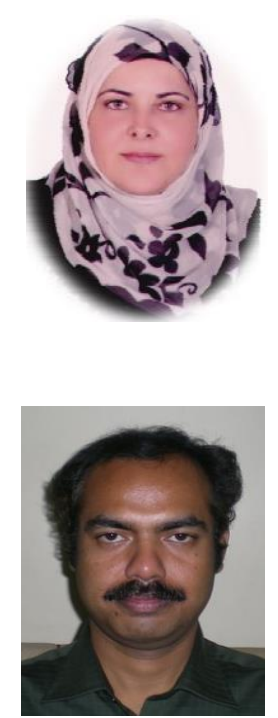

Wahiba Ben Abdessalem Karâa, completed her $\mathrm{PhD}$ in Computer Science, University of Paris, VII Jussieu. FRANCE. Currently she is an assistant professor at the High Institute of Management of Tunis. Dept. of Computer Science Applied to management. Tunisia. She has more than 50 research papers in various reputed journals and conferences.

Ruben Ray is an assistant professor of Department of IT, Government College of Engineering and Leather Technology, Kolkata, India. His research area includes Sensor networking and remote sensing.

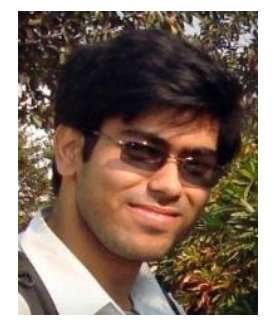

Shatadru Roy Chowdhury is a B.tech final year student of JIS College of Engineering, Kalyani, India. His major area in research is Digital Signal Processing Image analysis

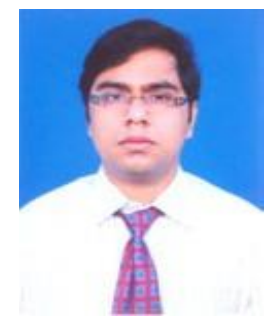

Sayan Chakraborty, is an M.Tech scholar of CSE department, JIS College of Engineering, Kalyani, India. He has around 18 research papers in various international journals and conferences.

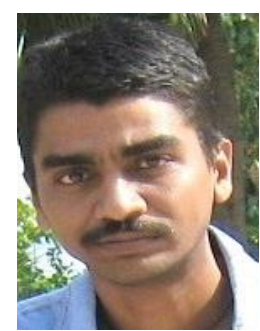

Nilanjan Dey, is a scholar of ETCE department, Jadavpur University, Kolkata, India. He is Visiting Scientist with Global Biomedical Technologies Inc., CA, USA. $\mathrm{He}$ is currently Asst. Professor in JIS College of Engineering, Kalyani, West Bengal, India. He has more than 80 research papers in various national \& international journals and conferences.

How to cite this paper: Subrata Nandi, Satyabrata Roy, Jayanti Dansana, Wahiba Ben Abdessalem Karaa, Ruben Ray, Shatadru Roy Chowdhury, Sayan Chakraborty, Nilanjan Dey,"Cellular Automata based Encrypted ECG-hash Code Generation: An Application in Inter human Biometric Authentication System", IJCNIS, vol.6, no.11, pp.1-12, 2014. DOI: $10.5815 /$ ijcnis.2014.11.01 